EPiC Series in Engineering
Volume 3, 2018, Pages 1350-1357
HIC 2018. 13th International
Conference on Hydroinformatics

\title{
Framework to Identify Optimal Configurations of (De)Centralised Wastewater Systems, in Abu Dis, West Bank
}

\author{
Alessia Matano $^{1}$, Peter Van Dee Steen ${ }^{2}$, Jawad Hassan ${ }^{3}$, Leonardo Alfonso ${ }^{2}$ \\ ${ }^{1}$ Technical University of Delft, Delft 2628CD, The Netherlands \\ ${ }^{2}$ IHE-Delft, Delft 2611AX, The Netherlands \\ ${ }^{3}$ Al Quds University, Abu Dis 89, Palestine \\ jamila.matano@gmail.com
}

\begin{abstract}
Decision-making processes for integrated wastewater management plans require the support of cost accounting and management techniques. This is particularly challenging in the Kidron - Wadi an-Nar basin, whose river is currently an open sewer and plans need to account for water stress, complex topography and socio-political differences. For these reasons, plans suggesting large centralised treatment facilities are difficult to implement. A potential solution, that can partially alleviate the problem, is the use of a number of smaller decentralised treatment facilities. The question that arises is, how to optimally configure combinations of centralised and decentralised wastewater treatment plants to achieve proper sanitation coverage in the basin and a sound water reuse? This study suggests a step forward towards solving the problem using a multi-objective optimisation framework. The objective functions considered are based on a Cost-Benefit analysis and the assessment of different wastewater treatment configurations. Sets of Pareto-optimal combinations of centralised and decentralised wastewater treatment solutions have been generated and evaluated in terms of the objective functions. The evaluation and comparison of wastewater treatment configurations include the potential reuses of the treated water. This analysis is especially essential in arid regions where limited water resources require an integrated and efficient water allocation.
\end{abstract}

\section{Introduction}

Integrated wastewater management plans get more and more attention worldwide. This is especially true in developing countries where economy and environment are sensitive issues. However, tools to provide an overview on the performance of different wastewater management configurations are limited. Few studies have specifically calculated the economic costs of wastewater treatment and 
reuse system through cost accounting and management techniques (Ruiz-Rosa, Garcia-Rodriguez, \& Mendoza-Jimenez, 2016).

A good wastewater planning and management not only offers the possibility to solve the issue related to the environmental pollution efficiently, but also gives the opportunity to add value to the area. Indeed, a wide picture of the possible combinations and the identification of solutions for reuse of the treated wastewater is essential in a primary analysis. In order to achieve this result, the investigation on the potential configurations and the identification of possible water reuses are essential in a preliminary analysis. Particularly in arid regions, the interest on wastewater reuse is increasing, to promote sustainable, efficient and appropriate water uses (Chu, Chen, Wang, \& Fu, 2004; Maksimovic, C. and Tejada-Guibert, J.A., 2001).

Currently, the lack in methods able to analyse efficiently different possibilities of planning, negatively affects the decision making process: no adequate preliminary elaborations are available to select the most suitable configurations. For this reason, potentially good solutions are not seen and therefore are not evaluated. This is true especially in particular contexts where an increasing number of variables and constraints play a role, increasing the complexity of the analysis.

Political fragmentation and water shortage make the town of Abu Dis (West Bank) an interesting study case for the development of a method for the optimisation of a wastewater management. This Palestinian town is located $3.8 \mathrm{~km}$ south-east of Jerusalem City, separated by a Segregation Wall that runs along its west boundary (The applied research institute, Jerusalem and AECID, 2012). The area presents a complex topography with a steep slope of around 20\%, which results in distinct drainage areas. Drinking water is provided by a local well and nonconventional source (purchased water sold by Gihoon, Israeli Company). Currently, the town experiments water shortage due to the low reliability of the sources related to the decrease of the groundwater table and technical and political issues. Additionally, due to political constrains, the urban development can take place only in limited areas, which leads to an increasing urban density and subsequent localised water demand. With a projection of a population increase of around $40 \%$ in the next five years (Abu Dis Local Council, 2017), an increase of the water shortage period is expected. In this situation, water reuse might be one of the few possibilities to satisfy the current and future need. Wastewater is also a topic of great concern in Abu Dis town. There are no sewage systems or wastewater plants that serve the area. Only cesspits are used to collect the wastewater, which are periodically emptied and discharged in the valley without any treatment. This study suggests a generic multi-objective optimisation framework using objective functions based on a Cost-Benefit analysis and assessed for different wastewater treatment configurations. To this end, a tool to efficiently evaluate all the components involved and their combinations is presented.

\section{Material and Methods}

\subsection{Optimisation problem}

The optimisation problem is formulated based on a Cost-Benefit analysis, taking into consideration the constraints of each configuration. A general formulation of the optimisation problem is: find the optimal number and type of wastewater treatment plants that maximise the benefits and the area of sanitation coverage and minimise the costs, taking into account available land, and water reuse possibilities. The benefit of a particular configuration can be expressed as: (Eq.1)

$B=\sum\left(b u_{i}+b a_{i}+c f_{i}+s f_{i}\right)$ 
where $\mathrm{B}$ is the total benefit of the configuration in analysis [ILS $] ; b u_{i}$ is the income from the sale treated water for urban purpose from WWTP $i$ [ILS]; $b a_{i}$ is the income from water sold for agriculture purpose from WWTP $i$ [ILS]. Finally, $c f_{i}$ and $s f_{i}$ are the income from the connection fees [ILS] and from the sanitation fees [ILS], respectively. (Matano', 2017)

The second objective function is the cost of the configuration (Eq. 2).

$$
C=\sum\left(\$ i n v_{i}+\$ o \& m_{i}+\$ \text { wnet }_{i}+R_{i}\right)
$$

where $C$ is the total cost of the configuration in analysis [ILS]; $\$ i n v_{i}$ is the investment cost of plant $i$, with capacity $Q_{i}$ [ILS]; $\$ o \& m_{i}$ is the O\&M cost of plant $i$, with capacity $Q_{i}[\mathrm{ILS}] ;$; wnet $_{i}$ is the cost of the sewage network [ILS] and $R_{i}$ is the cost of the reservoir related to the WWTP $i$ [ILS].

By combining Eq. 1 and Eq. 2, the Cost-Benefit ratio (C/B) is obtained, to be used as objective function to be minimised. Therefore, the optimisation problem can be formulated as:

$\min C / B=\min \frac{\sum\left(\$ i n v_{i}+\$ o \& m_{i}+\$ w n e t_{i}+R_{i}\right)}{\sum\left(b u_{i}+b a_{i}+c f_{i}+s f_{i}\right)}$

Subject to: jurisdiction boundaries, land use and water reuse possibilities.

\subsection{Framework and AOKP algorithm}

The proposed framework is composed by the Automatic Optimisation KidronNar Project (AOKP) algorithm, a Python-based script able to estimate Eq. (1) and Eq. (2) for any given set of WWTPs locations. The algorithm accepts any given number of sets to evaluate and stores the values of costs, benefits, coverage, among other parameters. The AOKP algorithm is divided into two main consecutive modules, namely the GIS analysis module and the computation module (Figure 1). The first module extracts and computes essential geospatial information related to area. The second module is the computation part, to estimate the objective functions of overall cost and benefit for any given configuration of WWTPs. This module includes the option of restricting the coverage area, in such a way that if a given set of WWTP locations does not comply with a predefined threshold coverage area, then such set is not accepted as solution. Both modules execute sub-modules: the watershed delineation and the join attribute by location in the GIS section and the Cost and Benefit estimation in the computation part. The input of the algorithm consist of GIS files with information about elevation, drainage network and built-up areas, to extract information about population density, land use, coverage and characteristics of the water drainage. The second module is set on input values with reference to an analysis of the wastewater treatment system in the West Bank. The AOKP algorithm can be used to evaluate two different treatment technologies, namely conventional activated sludge (CAS), and membrane bioreactor technology (MBR). When CAS is used, the agriculture reuse of the treated water is evaluated as part of the benefit. When MBR is used, the biological treatment process is made. For this technology, it is assumed that the treated water can be reused in the urban areas.

* ILS or Israeli New Shekel is the currency of Israel and is also used as a legal tender in the Palestinian territories of the West Bank and the Gaza Strip. 1 ILS corresponds to 0.24 euro. 


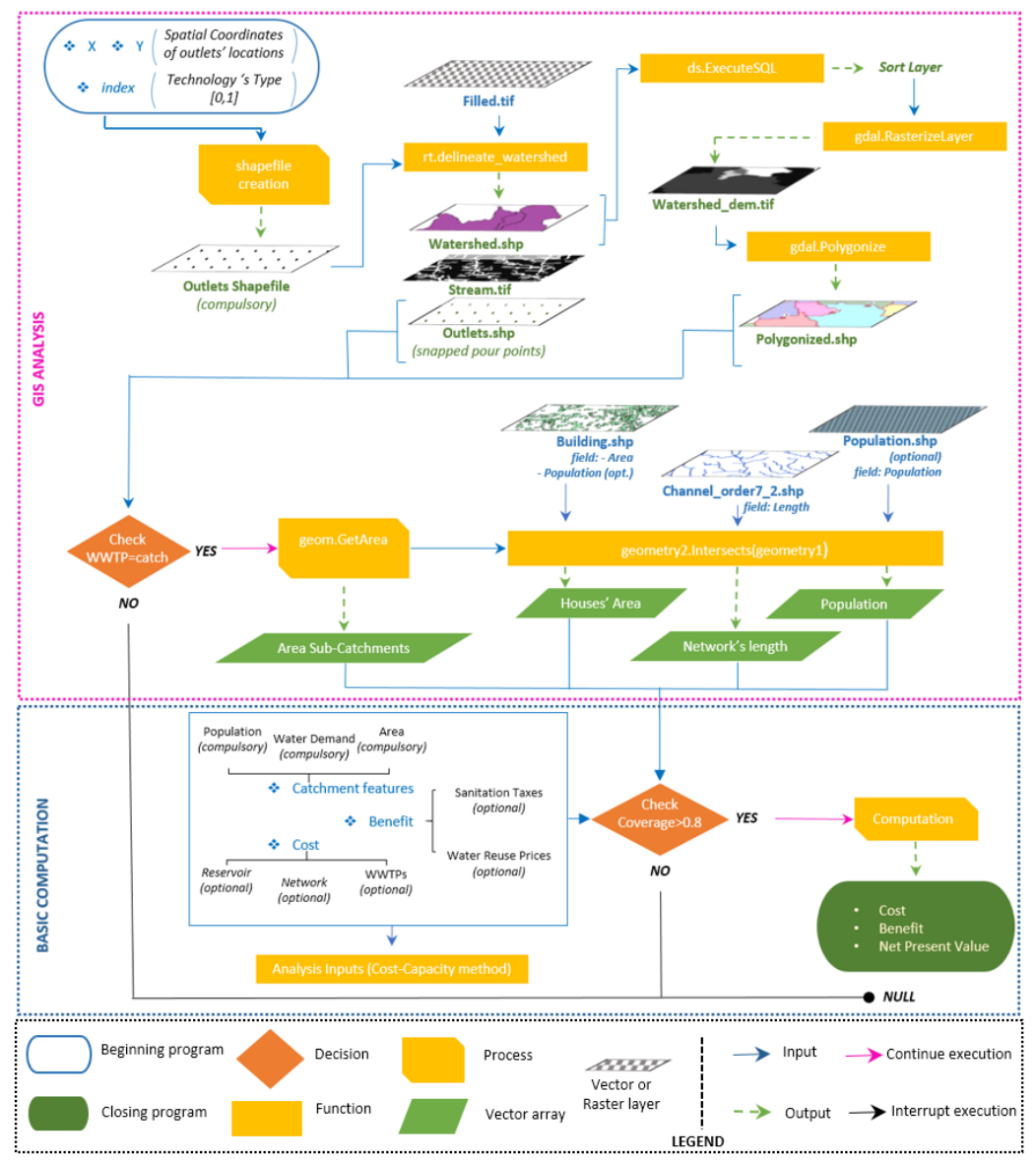

Figure 1: Scheme of the AOKP algorithm using a coverage area of $80 \%$

\section{Results and Discussion}

The AOKP algorithm was executed for combination of one, three and six WWTPs' locations, selected randomly from junction points of 4th order drainage network's branches according to the Strahler classification. Two types of analysis are provided next: 1) an analysis on the cost-benefit ratio for the two treatment technologies CAS and MBR with respect to coverage; 2) a multi-objective optimisation analysis based on cost-benefit ratio and coverage.

\subsection{Analysis based on treatment technology}

The cost-benefit ratio of configurations with CAS technology was plotted against the costbenefit ratio of those with MBR technology, for configurations including one, three and six WWTPs (see Figure 2) and for a coverage threshold of $80 \%$ (i.e. configurations that did not comply with at least $80 \%$ spatial coverage in terms of people served were not taken into account). From this analysis optimal solutions can be identified, corresponding to the minimum cost-benefit ratio value, highlighted by a dotted circle line in the graphs of Figure 2. Their corresponding configurations are plotted in the maps 
showed in Figure 4. It is possible to observe that the type of treatment technology adopted seems to have small influence on the optimal solution for one, three and six WWTPs. However, this optimal are strongly influenced by the spatial location of the WWTPs.
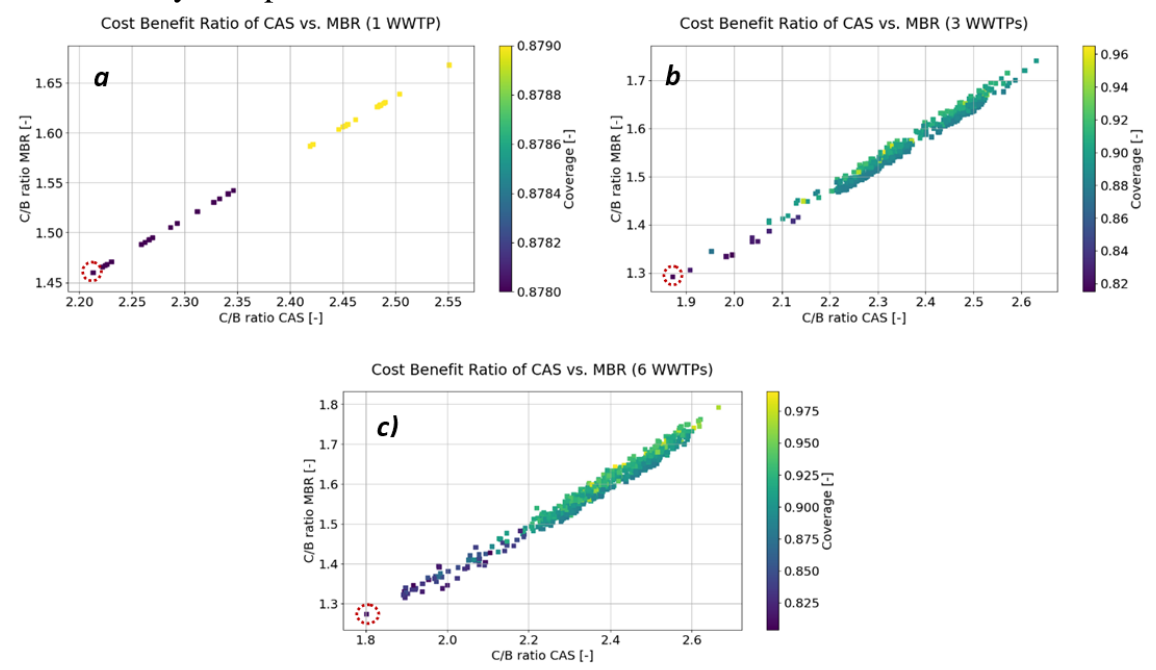

Figure 2: Cost-benefit ratio of CAS vs MBR with a minimum coverage of $80 \%$ for configurations including a) one WWTP; b) three WWTPs and c) six WWTPs

\subsection{Multi-objective optimisation analysis}

A multi-objective optimisation was carried to analyse cost-benefit ratio against coverage, which are conflicting objectives. The ideal solution would be a configuration that has total coverage and, simultaneously, a cost-benefit ratio of zero. Evidently, this solution does not exist. As the coverage increases, the costs also increase, which means that both objectives conflict with each other. To analyse this situation all the generated solutions were plotted on the objective function space, which allows for generating a Pareto frontier. As in any multi-objective optimisation procedure, the outcome of this framework is a set of possibly non-dominated solutions. This analysis was also carried out for configurations including one, three and six WWTPs, and for both CAS and MBR technologies. Additionally, in order to estimate the impact of the Israeli West Bank separation wall on the solutions, the configurations with at least one WWTP located beyond such wall were plotted in red (see Figure 3). This allowed to investigate the impact of the geo-political constrain on the optimal technical solutions. Below only the most significant results are shown. The Pareto analysis is showed only referring to configurations with CAS treatment process (Figure 3) as configurations with MBR process showed similar results. Additionally, a dotted circle line in the graphs highlight the solutions corresponding to the configurations in Figure 4.

Looking at Figure 3b, the configurations with at least one location beyond the segregation wall (light and dark red dots) have almost the same distribution of the other potential solutions. This is because the number of WWTPs is already high, compared to the size of the area under analysis and the presence or not of WWTPs beyond the wall does not affect prominently the cost and the coverage.

Instead, the analysis for configurations with three WWTPs shows slightly different results. Specifically, the distribution of the configurations with at least one location beyond the wall (light and dark red dots) seems again to follow the distribution for the other potential solutions (light and dark blue dots) (Figure 3a). 

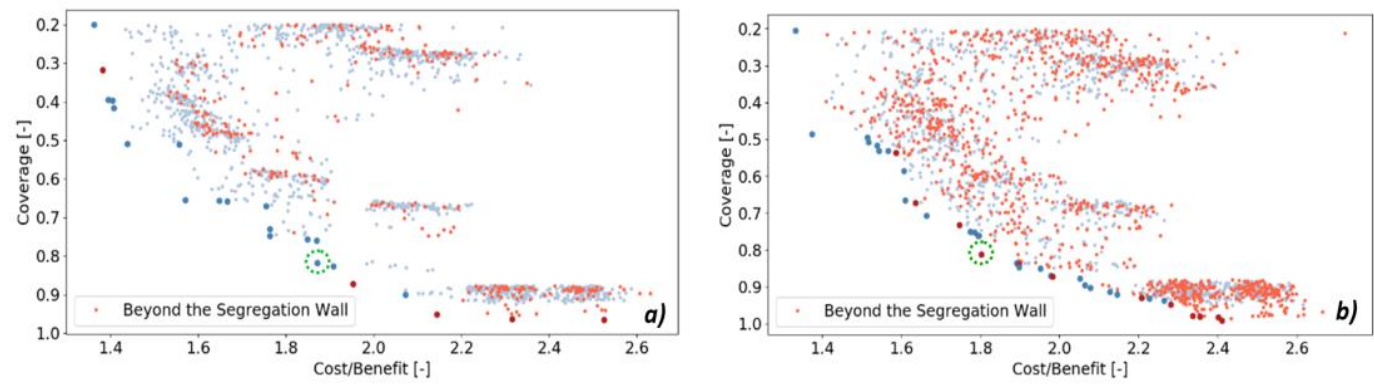

Figure 3: Pareto Analysis for CAS process and minimum coverage of 20\% (the solutions on the Pareto Front are showed by bigger and darker dots). a) three WWTPs b) six WWTPs

However, a marked arrangement of the points on linear trends can be observed; without differences among the locations of the WWTPs, the solutions tend to gather in areas scattering along the $\mathrm{x}$-axis. This can be explained by the distribution of the built-up areas that are not homogeneously spread in the analysed area. For this reason, different configurations have the same coverage but with different overall costs according to the distance to the served houses. Another difference from the previous graph arises from looking at the dark dots of the Pareto front. The latter is mostly defined by potential configurations with one WWTP located beyond the wall (dark red dots). This means that the best technical solutions conflicts with the geo-political constrain. This emerges only with configurations of three WWTPs and not six, due to the altimetry of the municipal area investigated. Specifically, almost all the area belongs to only one catchment that drains towards the lowest part of the municipality. However, on the west side, a small part of the municipality belongs to another catchment, where the segregation wall is located. This means that the highest coverage that can be reached is with one or two WWTPs located in the downstream part of the river and one additional WWTP located just beyond the wall, in order to connect the few houses that drain to the west side.

In the technology-based analysis, the optimal sanitary coverage for Abu Dis town can be identified through the comparison of different combinations of WWTPs (one, three, and six WWTPs). However, it will be always stressed that the optimum identified is not absolute as the search space is much bigger than the space that was explored by the random search.

Analysing Figure 5, the configuration of six WWTPs is the one corresponding to the minimum values of the cost-benefit ratio for both treatment processes. In the multi-objective optimisation analysis, a set of optimal solutions along the Pareto frontier are identified. These can be subsequently used by decision makers for selecting a preferred wastewater management plan for implementation, according to the available budget and the main goals of the project.

In Figure 6, the non-dominated points which are members of the Pareto set are shown. Solutions with MBR treatment technology are closer to the axes, compared to the solutions with CAS treatment technology. This clearly shows treatment plants with MBR technologies offer better coverages for the same costs as CAS. The comparison of optimal Pareto fronts shows that optimal solutions can be identified among the configurations with MBR treatment technology. This result differs from what come out from the cost-benefit analysis of the treatment technologies carried out in the corresponding section. Specifically, by only looking at the cost-benefit ratio, the optimal solution is identified among the configurations with six WWTPs, and no influence of the treatment process on the configurations rise. However, if also the coverage is introduced as objective to be optimized, the configurations with MBR become the best technical option for satisfying both objectives. 

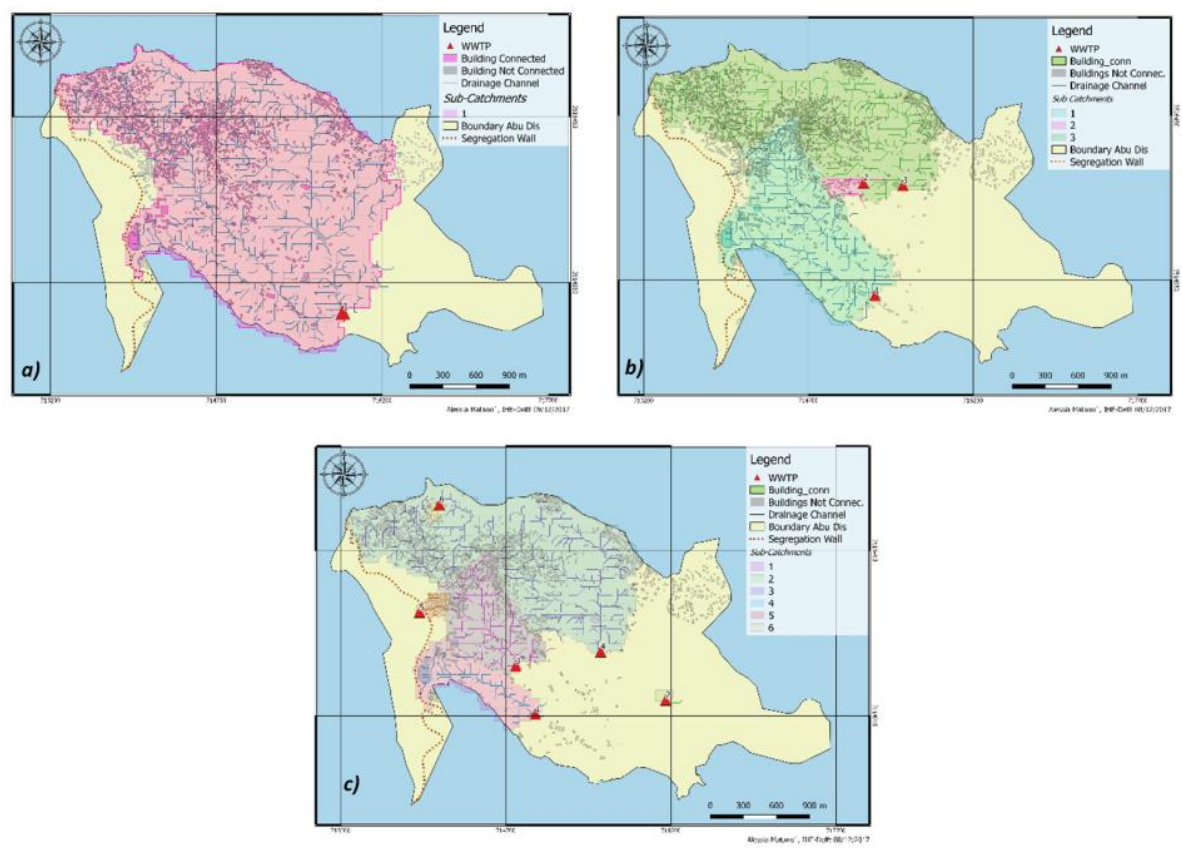

Figure 4: Optimal configurations according to the minimum Cost-Benefit ratio. a) configuration with one WWTP b) with three WWTPs and c) with six WWTPs

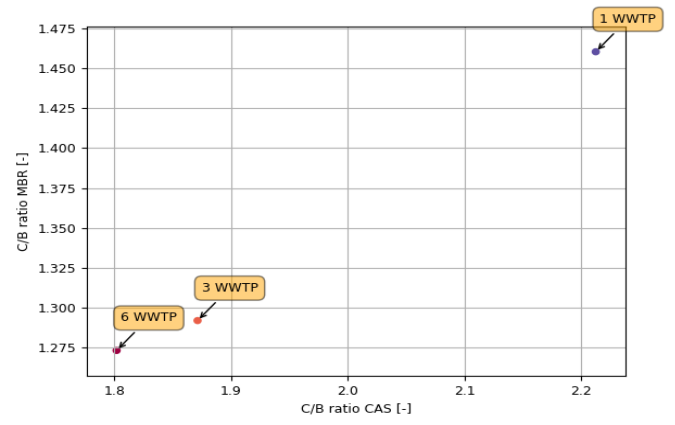

Figure 5: Cost-benefit ratio of the optimal configurations with one, three, six WWTPs (limit coverage: $80 \%$ )

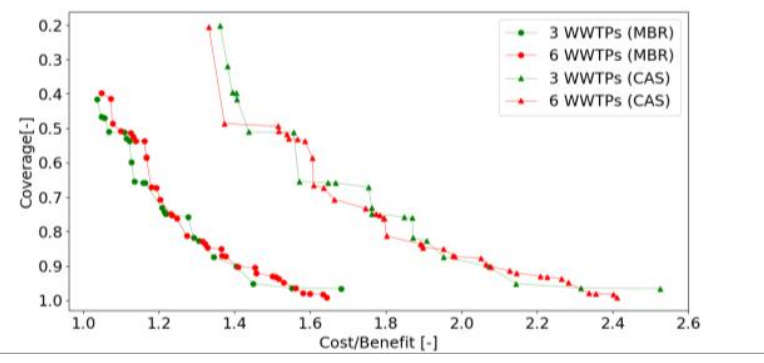

Figure 6: Pareto set for combinations of three (dotted lines) and six (continuous lines) WWTPs, for CAS (triangle marker) and MBR (circled marker) treatment technologies (minimum coverage equal to 20\%) 


\section{Conclusion}

In this study, a generic multi-objective optimisation framework with objective functions based on a cost-benefit analysis was performed for different wastewater treatment configurations. The developed methodology offers a relevant support in the decision making process. This is especially true in the Palestinian Territories where different plans have been developed, but have not been implemented. This has led to the adoption of disconnected projects, which miss a more general overview of the problem under investigation. The prohibitive topography is also another obstacle to the planning process that does not allow to follow political boundaries in order to identify the area covered by each WWTP. Additionally, the lack of communication and sharing information among stakeholders and fragmented division of the area due to social-political conflicts, make even more complicated the design of an integrated water management plan.

The (de)centralised configuration could partially address this challenges and the current study can offer a significant support in this analysis. The proposed framework is able to give a glance of the needs of the area as well as an evaluation of possible configurations. Specifically, it offers a clear view of the repartitions of the served areas according to their natural drainage catchments. Looking at larger scale and at the natural watershed, possible solutions for a wastewater management are more easily underlined. This tool is able not only to identify an optimal subdivision of the area, but also investigate potential reuses of the treated water. Moreover, geopolitical constraints can be easily included .

The developed algorithm is robust and fast, and it can be applied to any other study case, provided the input data is available. Moreover, the parameters can easily be changed to adjust the objective functions, including for example weight factors for each of their terms. AOKP has so far been applied on a small scale and needs to be extended to a larger scale. We believe that this will further improve the performance of the model. Indeed, the algorithm depends on generic design and cost-benefit parameters which results cannot replace detailed engineering decisions on the ground.

In the present study, random configurations were generated and run by the algorithm as a first optimisation approach. However, the procedure has been designed in a way that it is easy to connect to any evolutionary optimisation algorithm, opening the door to a set of interesting studies, including the evaluation of the effect of the restriction of different political boundaries, the combination of treatment technologies in one solution set and the optimal expansion of the configuration for future developments. More advanced studies that can be carried out are, for instance, the uncertainty analysis of the parameters and the sensitivity of each term of the objective functions.

\section{References}

Abu Dis Local Council. (2017). Municipality Development Plan.

Chu, J., Chen, J., Wang, C., \& Fu, P. (2004). Wastewater reuse potential analysis: Implications for China's water resources management. Water Research, 38(11), 2746-2756. https://doi.org/10.1016/j.watres.2004.04.002

Maksimovic, C. and Tejada-Guibert, J.A. (2001). The challenge of urban water management. Frontiers in Urban Water Management, IWA.

Matano`, A. (2017). Framework to Identify Optimal Configurations of ( De ) Centralised Wastewater Systems, in Abu Dis, West Bank.

Ruiz-Rosa, I., Garcia-Rodriguez, F. J., \& Mendoza-Jimenez, J. (2016). Development and application of a cost management model for wastewater treatment and reuse processes. Journal of Cleaner Production, 113, 299-310. https://doi.org/10.1016/j.jclepro.2015.12.044

The applied research institute, Jerusalem and AECID, S. cooperation. (2012). Abu Dis Town Profile. 